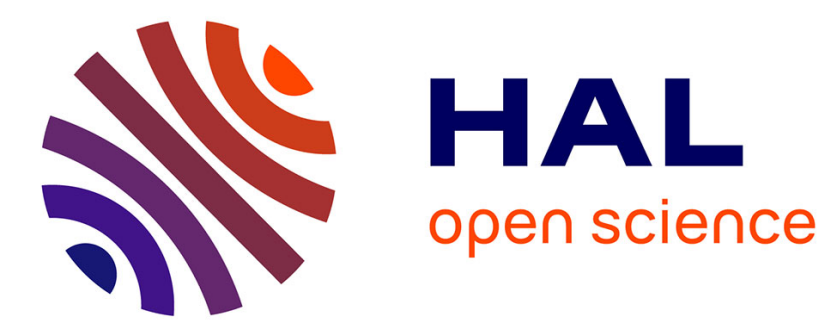

\title{
Tensor network simulation of polaron-polaritons in organic microcavities
}

Javier Del Pino, Florian A. Y. N. Schröder, Alex Chin, Johannes Feist, Francisco J. Garcia-Vidal

\section{To cite this version:}

Javier Del Pino, Florian A. Y. N. Schröder, Alex Chin, Johannes Feist, Francisco J. Garcia-Vidal. Tensor network simulation of polaron-polaritons in organic microcavities. Physical Review B: Condensed Matter and Materials Physics (1998-2015), 2018, 98 (16), 10.1103/PhysRevB.98.165416 . hal02372598

\section{HAL Id: hal-02372598 https://hal.sorbonne-universite.fr/hal-02372598}

Submitted on 20 Nov 2019

HAL is a multi-disciplinary open access archive for the deposit and dissemination of scientific research documents, whether they are published or not. The documents may come from teaching and research institutions in France or abroad, or from public or private research centers.
L'archive ouverte pluridisciplinaire HAL, est destinée au dépôt et à la diffusion de documents scientifiques de niveau recherche, publiés ou non, émanant des établissements d'enseignement et de recherche français ou étrangers, des laboratoires publics ou privés. 


\title{
Tensor network simulation of polaron-polaritons in organic microcavities
}

\author{
Javier del Pino, ${ }^{1, *}$ Florian A. Y. N. Schröder, ${ }^{2}$ Alex W. Chin,,${ }^{3,2}$ Johannes Feist, ${ }^{1}$ and Francisco J. Garcia-Vidal ${ }^{1,4}$ \\ ${ }^{1}$ Departamento de Física Teórica de la Materia Condensada and Condensed Matter Physics Center (IFIMAC), \\ Universidad Autónoma de Madrid, E-28049 Madrid, Spain \\ ${ }^{2}$ Cavendish Laboratory, University of Cambridge, J. J. Thomson Avenue, Cambridge, CB3 OHE, United Kingdom \\ ${ }^{3}$ Institut des NanoSciences de Paris, Sorbonne Université, 4 place Jussieu, boîte courrier 840, 75252, PARIS Cedex 05 \\ ${ }^{4}$ Donostia International Physics Center (DIPC), E-20018 Donostia/San Sebastián, Spain
}

(Received 2 July 2018; revised manuscript received 26 September 2018; published 12 October 2018)

\begin{abstract}
In the regime of strong coupling between molecular excitons and confined optical modes, the intramolecular degrees of freedom are profoundly affected, leading to a reduced vibrational dressing of polaritons compared to bare electronically excited states. However, existing models only describe a single vibrational mode in each molecule, while actual molecules possess a large number of vibrational degrees of freedom and additionally interact with a continuous bath of phononic modes in the host medium in typical experiments. In this work, we investigate a small ensemble of molecules with an arbitrary number of vibrational degrees of freedom under strong coupling to a microcavity mode. We demonstrate that reduced vibrational dressing is still present in this case, and show that the influence of the phononic environment on most electronic and photonic observables in the lowest excited state can be predicted from just two collective parameters of the vibrational modes. Besides, we explore vibrational features that can be addressed exclusively by our extended model and could be experimentally tested. Our findings indicate that vibronic coupling is more efficiently suppressed for environments characterized by low-frequency (sub-Ohmic) modes.
\end{abstract}

DOI: 10.1103/PhysRevB.98.165416

\section{INTRODUCTION}

When an ensemble of quantum emitters interacts with a confined electromagnetic field (EM) mode, the system can enter into the strong coupling regime, resulting in the formation of light-matter quasiparticles known as polaritons, which inherit properties from each constituent. In particular, organic molecules present favorable features to achieve large couplings to optical modes at room temperature, due to their tightly bound Frenkel excitons [1,2] with large dipole moments. These properties offer an optimal experimental platform for polariton lasing [3,4], enhanced exciton conductivity [5], light harvesting [6], and suppression of photobleaching in $J$ aggregates [7]. The strong light-matter coupling regime can be attained experimentally in a variety of different setups, such as in microcavities filled with a large number of molecules [8] or, more recently, in nanoscale plasmonic resonators coupled to just a few molecules $[9,10]$.

The formation of exciton-polaritons in inorganic semiconductor microcavity systems [11] is known to decouple polariton states from the phonons, which act uniquely on the matter component. In this case, the bare exciton-phonon coupling is already relatively weak and can be treated perturbatively by means of an effective scattering rate for the extended inorganic polariton states that enables to treat their nonequilibrium dynamics with simple kinetic models [11-14]. For coupling to acoustic phonons, the very steep dispersion (low density of final scattering states) in the lower-polariton branch leads to

\footnotetext{
*francisco.delpino@uam.es
}

a very effective decoupling from the phononic environment and inefficient thermalization of polaritons [11-14]. The existence of spectrally resolved exciton-polaritons in the presence of the phonons could also be addressed in simple models of coupled damped harmonic oscillators that introduce the effect of phononic dephasing on the formation of polariton modes $[11,15]$.

In contrast to inorganic systems, organic microcavities have only recently emerged as a subject of investigation, with several works predicting the reduction of vibrational displacement (RVD) in exciton-polariton states compared to bare-molecule excited states with significant implications in the chemical reactivity of such molecules, such as polaritonicbased catalysis of electron transfer [16] and photoisomerization [17]. Unlike inorganic systems, organic materials display very strong and structured exciton-vibrational coupling arising from the intramolecular modes of the chromophores. Quantum correlation effects, such as polaron formation, must be included, as must the role of non-negligible reorganization energy and the possible impact of having comparable lightmatter and vibronic interactions in the real-time dynamics and steady states. Approaches typically rely on the Holstein-TavisCummings (HTC) model, which describes a single EM mode coupled to a collection of molecules with a single vibrational mode each [16,18-21]. Nevertheless, actual molecules possess a large number of nuclear oscillation modes and, additionally, interact with a continuous bath of phononic modes in the host solvent [22] or polymer matrix [23] in typical experiments, which induces additional chemical changes [24]. In this case, the persistence of the RVD in polaritons is unknown. 


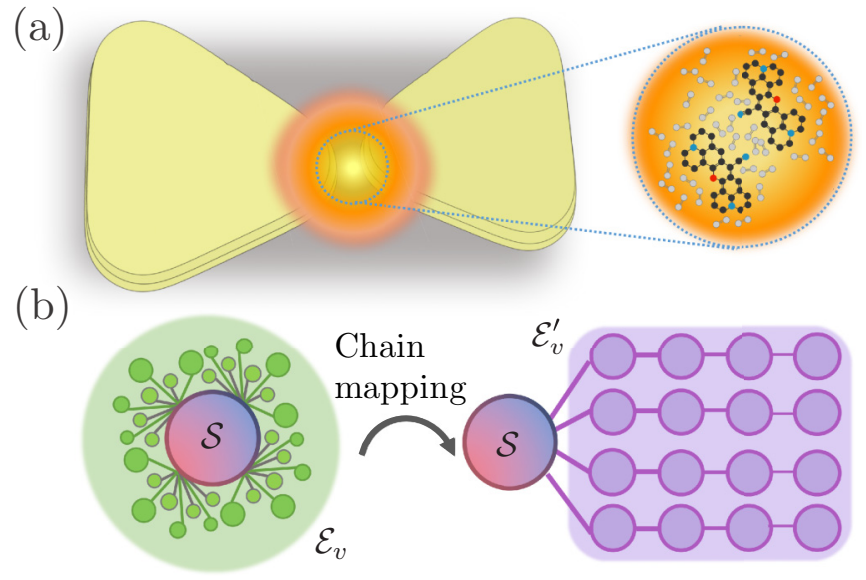

FIG. 1. (a) Sketch of a molecular ensemble interacting with a confined EM resonance (dashed region) and with the host environment (gray circles). (b) Scheme illustrating the mapping of the vibrational modes yielding the multichain Hamiltonian used in the simulations, where the excitons interact with collective reaction coordinates in the molecules.

In this work, we present a study of the lowest-energy polaritonic state supported by a small ensemble of organic molecules under strong coupling to an optical microcavity mode, sketched in Fig. 1(a). Under the assumption that the molecules are close to their mechanical equilibrium, we include the whole vibrational spectrum of nuclear and environmental modes in our analysis, specified by the spectral density $J_{v}(\omega)$. The level of modeling of the molecules, therefore, goes beyond the HTC Hamiltonian, but still enables exploration of the full many-body wave function employing canonical bosonic operators and thus the toolbox from quantum optics. Due to its mixed threefold photonic-excitonic-phononic character, this eigenstate is dubbed the lower polaron-polariton (LPP), adopting the nomenclature in Ref. [25]. To treat phononic and photonic processes in the molecules in the same footing, and, at the same time, deal with an arbitrary number of vibrations, we exploit a tensor network (TN) representation of the system's wave function that extends the widespread matrix product states (MPS) for one-dimensional quantum chains [26]. This means the LPP is retrieved by variational minimization of the $\mathrm{TN}$ via the variational matrix product state (VMPS) algorithm [27], which filters out the most relevant components mixed in the state capturing their mutual entanglement.

Our results show the robustness of RVD in the LPP wave function beyond the HTC model, extending its validity to arbitrary structured phononic baths. Moreover, the trend for larger ensembles indicates the effective suppression of polaron formation in the thermodynamic limit. Intriguingly, we observe that excitonic and photonic components are largely independent of specific molecular details, and can be reproduced by an effective HTC model determined by two singlemolecule cumulative parameters. Specifically, these are the reorganization energy $\Delta$ [28], associated with the reequilibration of the vibrational modes after electronic excitation, and the mean phononic oscillation frequency $\Omega_{v}$ that corresponds to a collective reaction coordinate. Conversely, the LPP vibrational properties are strongly molecule dependent and thus shaped by $J_{v}(\omega)$.

The paper is organized as follows: In Sec. II we first present the model and introduce the TN based algorithm aimed to target the LPP wave function. Namely, we analyze in Sec. III the LPP eigenfrequency and the excitonic and photonic states reduced populations as a function of vibronic coupling, including the vibration-free polaritonic components mixed into the state. In Sec. IV focus on the "spectrum" of vibrational displacement as the spectral density is varied. Finally, in Sec. V we test our conclusions for organic molecules with a highly structured spectral density, using rhodamine 800 as a test case.

\section{MODEL AND METHODS}

Our model includes a collection of $N$ identical molecules, containing a single exciton with frequency $\omega_{e}$ and ladder operators $\hat{\sigma}_{ \pm}^{(i)}(i=1, \ldots, N)$, placed within the volume of a nanocavity or microcavity supporting a single dispersionless EM mode (frequency $\omega_{O}=\omega_{e}$ ) and annihilation operator $\hat{a}$. The total Hamiltonian contains two different parts, as schematically depicted in Fig. 1(b). First, the system $\mathcal{S}$ that accounts for the excitons within the molecules, the cavity EM mode, and their mutual coupling, measured by the collective Rabi frequency $\Omega_{R}$ and treated within the rotating-wave approximation (setting $\hbar=1$ )

$$
\hat{H}_{\mathcal{S}}=\omega_{O} \hat{a}^{\dagger} \hat{a}+\sum_{i=1}^{N} \omega_{e} \hat{\sigma}_{+}^{(i)} \hat{\sigma}_{-}^{(i)}+\frac{\Omega_{R}}{2 \sqrt{N}} \sum_{i=1}^{N}\left(\hat{a}^{\dagger} \hat{\sigma}_{-}^{(i)}+\hat{\sigma}_{+}^{(i)} \hat{a}\right) .
$$

We neglect interexcitonic interactions, which we assume are screened out by the host environment. In the singleexcitation subspace, $\hat{H}_{\mathcal{S}}$ is exactly solvable. Its eigenstates are two polaritons, upper (UP) and lower (LP), $| \pm\rangle=\left(\hat{a}^{\dagger}|G\rangle \pm\right.$ $|B\rangle) / \sqrt{2}$, with frequencies $\omega_{ \pm}=\omega_{O} \pm \Omega_{R} / 2$, which result from the hybridization of the collective excitonic bright state $|B\rangle=\left(\sum_{i=1}^{N} \hat{\sigma}_{+}^{(i)}|G\rangle\right) / \sqrt{N}$ with the cavity EM mode (here $|G\rangle$ stands for the global vacuum state). In addition, there are $(N-1)$ so-called dark states (DS), $|d\rangle \in \mathcal{D}$, which are purely excitonic states of frequency $\omega_{e}$ that are orthogonal to $|B\rangle$.

The second part of the Hamiltonian describes the vibrational subspace $\mathcal{E}_{v}^{(i)}$ containing a large number $M_{v}$ of vibrational modes both inside the molecule and in the host environment, and their elastic coupling to the excitons. The $k$ th vibrational mode is approximated by a harmonic oscillator of frequency $\omega_{k}$ (valid close to the equilibrium position) with annihilation operator $\hat{b}_{k}^{(i)}$ and exciton-phonon coupling strength $\lambda_{k}^{(i)}$ :

$$
\hat{H}_{v}=\sum_{i=1}^{N} \sum_{k=1}^{M_{v}} \omega_{k}^{(i)} \hat{b}_{k}^{\dagger(i)} \hat{b}_{k}^{(i)}+\sum_{i=1}^{N} \sum_{k=1}^{M_{v}} \lambda_{k}^{(i)}\left(\hat{b}_{k}^{(i)}+\hat{b}_{k}^{\dagger(i)}\right) \hat{\sigma}_{+}^{(i)} \hat{\sigma}_{-}^{(i)} .
$$

The properties of these modes, $\left\{\omega_{k}^{(i)}, \lambda_{k}^{(i)}\right\}$, are encoded in the spectral density $J_{v}^{(i)}(\omega)=J_{v}(\omega)=\pi \sum_{k=1}^{M_{v}} \lambda_{k}^{2} \delta\left(\omega-\omega_{k}\right)$. Here, the exciton-phonon coupling does not lead to nonradiative decay [24]. The coupling parameters $\lambda_{k}$ describe the 
relative shifts of electronic potential surfaces between ground and excited states. For the uncoupled molecules $\left(\Omega_{R}=0\right)$, the total Hamiltonian $\hat{H}_{\mathcal{S}}+\hat{H}_{v}$ becomes a sum of exactly diagonalizable independent-boson models [29]. Namely, the eigenexcitations or Lang-Firsov polarons can be visualized as localized "phonon clouds" surrounding the excitons at each molecule: $\left|D_{i}\right\rangle=e^{-\hat{S}_{i}}|E\rangle$, with $\hat{S}_{i}=\hat{\sigma}_{+}^{(i)} \hat{\sigma}_{-}^{(i)} \sum_{k} \frac{\lambda_{k}^{(i)}}{\omega_{k}^{(i)}}\left(\hat{b}_{k}^{(i)}-\right.$ $\left.\hat{b}_{k}^{(i) \dagger}\right)$, where $|E\rangle=\hat{\sigma}_{+}^{(i)}|G\rangle$.

For typical molecules, when $M_{v}$ becomes large [or even formally infinite when a continuum approximation for $J_{v}(\omega)$ is used], most standard approaches of quantum optics to calculate the eigenstates of the system fail, and even many approximate methods become prohibitively expensive. For instance, direct diagonalization of the total Hamiltonian is unfeasible, even for $N=1$. However, a VMPS algorithm permits to calculate the full wave function including all degrees of freedom of either the time-evolved wave function after excitation (analyzed in [30]) or the ground state within the single electronic excitation subspace. This is precisely the "lower polaron-polariton" [16,20,25] analyzed in the socalled Holstein-Tavis-Cummings (HTC) model [16,20,21,25], which deals with a single vibrational mode with frequency $\omega_{v}$ and vibronic coupling $\lambda_{v}$, i.e., $J_{\mathrm{HTC}}(\omega)=\pi \lambda_{v}^{2} \delta\left(\omega-\omega_{v}\right)$. In particular, the Hamiltonian (2) extends the HTC to an arbitrary number $M_{v}$ of vibrational modes.

Here, we employ a tensor network state based approach that is numerically exact as long as convergence is reached, permitting a nonperturbative exploration of the quantum effects arising in the LPP. It does not rely on any specific form of the spectral density and, in particular, can be used both for discrete and continuous $J_{v}(\omega)$. To apply this method, we perform an orthogonal mapping of the modes in the $N$ vibrational (green in Fig. 1) environments $\left(\mathcal{E}_{v}^{(i)}\right)$. We rely on the chain transformation introduced in [31], which maps the molecular Hamiltonian (2) for an exciton coupled to many independent vibrations to an exciton coupled only to the first site in a chain of coupled oscillators, leading to the vibrational Hamiltonian $\hat{H}_{v}^{\prime}=\sum_{i=1}^{N} \hat{H}_{v}^{\prime(i)}$ :

$$
\begin{aligned}
\hat{H}_{v}^{(i)}= & \Omega_{v} \hat{c}_{0}^{(i) \dagger} \hat{c}_{0}^{(i)}+\eta \hat{\sigma}_{+}^{(i)} \hat{\sigma}_{-}^{(i)}\left(\hat{c}_{0}^{(i)}+\hat{c}_{0}^{(i) \dagger}\right) \\
& +\sum_{l=1}^{M_{v}-1} \omega_{l} \hat{c}_{l}^{(i) \dagger} \hat{c}_{l}^{(i)}+\sum_{l=0}^{M_{v}-2} t_{l}\left(\hat{c}_{l}^{(i) \dagger} \hat{c}_{l+1}^{(i)}+\hat{c}_{l+1}^{(i) \dagger} \hat{c}_{l}^{(i)}\right) .
\end{aligned}
$$

This transformation results in the star coupling structure for $\hat{H}_{\mathcal{S}}+\hat{H}_{v}^{\prime}$ sketched in the right part of Fig. 1(b). The modes are thus regrouped in chains with length $L=M_{v}$ [32], with only the first mode coupled to the exciton-photon subspace $\mathcal{S}$ (red-blue). Namely, each exciton is coupled to a single collective reaction mode defined by $\eta \hat{c}_{0}=\sum_{k} \lambda_{k} \hat{b}_{k}$ [33-36], with total amplitude

$$
\eta=\sqrt{\int_{0}^{\omega_{c}} J_{v}(\omega) d \omega / \pi},
$$

which generalizes the vibronic coupling in the HTC model ( $\eta=\lambda_{v}$ in this case) and average bath frequency

$$
\Omega_{v}=\frac{\int_{0}^{\omega_{c}} \omega J_{v}(\omega) d \omega}{\int_{0}^{\omega_{c}} J_{v}(\omega) d \omega},
$$

which similarly generalizes $\omega_{v}$. All other chain modes become connected through nearest-neighbor hopping interactions $t_{l}$. The discussion below shows an important quantity to characterize the vibronic coupling in the system in the so-called reorganization energy, given by

$$
\Delta=\frac{1}{\pi} \int_{0}^{\omega_{c}} \frac{J_{v}(\omega)}{\omega} d \omega,
$$

and directly linked with the Stokes (frequency) shift between maxima of emission and absorption spectra in organic molecules, given by $2 \Delta[37,38]$.

The star Hamiltonian (3) permits direct implementation of the VMPS algorithm. To this end, the LPP wave function $\left|\psi_{-}\right\rangle$is represented by a tensor network with maximum bond dimensions $D$, which directly mimics the coupling structure in the star Hamiltonian [Fig. 1(b)], making the representation numerically efficient. This procedure, coupled with a variational approach to calculate the LPP, leads to a multichain variant of the density matrix renormalization group algorithm (DMRG) for 1D quantum lattices [39]. When continuous vibrational spectra are taken into account, the formally infinite phononic chains are truncated at length $L$, which must be chosen large enough to reach convergence. A more detailed discussion can be found in the Appendices A 1 and A 2 .

We present results for a maximum of $N=5$ molecules. For large molecular ensembles, a severe memory bottleneck occurs if the system $\mathcal{S}$ is stored in a single root tensor leading to exponential scaling in $N$. However, as shown in [40,41], it would be possible to restore the efficiency of the numerical method while maintaining precision, by further decomposing the root node into a tree TN, where each final branch represents an exciton or the cavity photon and is coupled to a single chain. Developing a suitably efficient tree model requires an explicit analysis of "entanglement topology" of the state, an idea that has recently been implemented to allow the simulation of multienvironment linear vibronic models constructed from ab initio parametrizations of small molecules [42]. We here focus on the intrinsic properties of the ground state within the single excitation subspace alone but note that the same approach also allows efficient simulation of the polariton time evolution, which provides insight on the highly nontrivial dissipative processes into the LPP and their impact on the spectral linewidths in linear response [30]. To further ameliorate memory issues for large chain mode occupations, we employ an optimal boson basis for the chain tensors [43], which can be determined on the fly via VMPS [44]. A more detailed description of the theoretical approach can be found in [44].

\section{A. Holstein-Tavis-Cummings limit}

In order to connect our study with the cases available in the literature, we start by briefly revisiting the HTC model, first introduced by Kirton et al. [18], which can be solved by various methods, including direct numerical diagonalization and via variational Ansätze [16,21,25]. Vibronic coupling is parametrized by the reorganization energy $\Delta=\lambda_{v}^{2} / \omega_{v}$, obtained from Eq. (6). In Figs. 2 and 3 we show the eigenenergies of the system for $N=1$ and 2 molecules, with a single electronic/optical excitation in the system at maximum. 


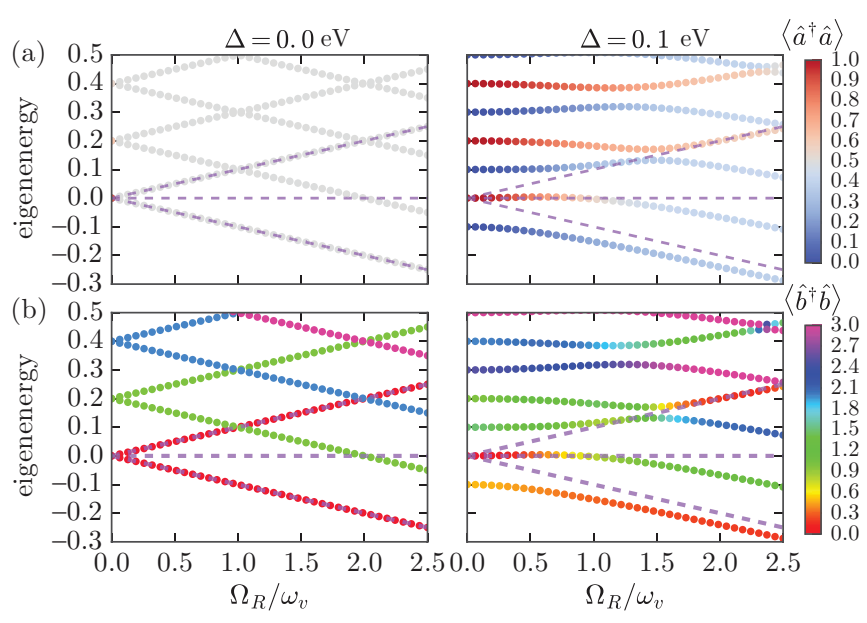

FIG. 2. (a) Photonic components in the eigenspectrum of the HTC model for a single molecule as a function of the ratio between Rabi frequency $\Omega_{R}$ and vibrational frequency $\omega_{v}$. (b) Vibrational component. Dashed purple lines indicate the upper and lower vibration-free polariton energies. The energy origin is set at $\omega_{e}=\omega_{O}=0$.

The coexistence of vibronic and photonic couplings results in an involved eigenspectrum where states are characterized by a triple mixture of vibrational, photonic, and excitonic states $[16,45]$. In these figures, the color scale is used for displaying the cavity and vibrational populations $\left\langle\hat{a}^{\dagger} \hat{a}\right\rangle$ and $\left\langle\hat{b}^{\dagger} \hat{b}\right\rangle=\sum_{i}\left\langle\hat{b}^{(i) \dagger} \hat{b}^{(i)}\right\rangle$, respectively.

For a single molecule, the "vibration-free" eigenstates (i.e., for $\Delta=0$ ) system are precisely the polaritons described in left panels of Fig. 2, together with their vibrational sidebands corresponding to excited molecular phonons. The energies of these states are, therefore, $\omega_{n}^{ \pm}=\omega_{ \pm}+n \omega_{v}$, $n \in \mathbb{N}$. Conversely, if there is no coupling with the cavity

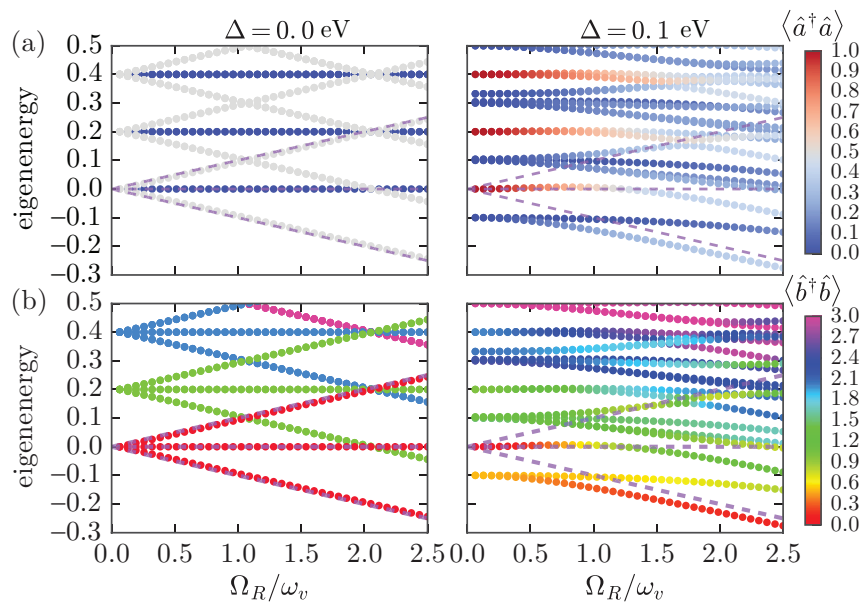

FIG. 3. (a) Photonic components in the eigenspectrum of the HTC model for two molecules as a function of the ratio between Rabi frequency $\Omega_{R}$ and vibrational frequency $\omega_{v}$. (b) Total vibrational components $\left\langle\hat{b}^{\dagger} \hat{b}\right\rangle=N\left\langle\hat{b}^{(i) \dagger} \hat{b}^{(i)}\right\rangle$. Dashed purple lines indicate the upper and lower vibration-free polariton and excitonic dark-state energies. The energy origin is set at $\omega_{e}=\omega_{O}=0$, and we consider $\omega_{v}=0.2 \mathrm{eV}$. mode (limit $\Omega_{R}=0$ ), vibronic coupling results in the formation of a polaron with energy $\omega_{e}-\Delta$. For intermediate energy scales (right panels), the ground state in the singleexcitation space (LPP) has a partially polaritonic nature [25]. In Fig. 2(a), higher-energy polaritonic sidebands display anticrossings caused by vibronic interaction when resonance conditions are met. This is confirmed by the fact that eigenstates do not have a well-defined phonon number in the vicinities of the split regions [see Fig. 2(b)]. Also, for large $\Delta$, the photonic nature of the states becomes transferred to higher-energy excitations as it becomes increasingly unfavorable to form hybrid light-matter quasiparticles in the system, while for higher $\Omega_{R}$ the states contain a greater fraction of the "original" polaritons. As a minimum extension to the collective behavior of the system, we analyze in the following a molecular dimer within the HTC model. In this case, the presence of one dark excitonic state $|d\rangle=\left(\hat{\sigma}_{+}^{(1)}-\hat{\sigma}_{+}^{(2)}\right)|G\rangle / \sqrt{2}$ besides vibrational sidebands (frequencies $\omega_{d}^{n}=\omega_{e}+n \omega_{v}, n \in \mathbb{N}$ ) is also present in the vibration-free eigenstates displayed in the left panels of Fig. 3. Vibronic interaction results in a larger shift of dark states as compared to polaritons, as inferred from the right panels in Fig. 3. Eigenenergies present actual crossings in addition to anticrossings, leading to a much richer structure of eigenstates than for $N=1$ [45]. Comparing the vibrational components in Fig. 3(b) with those of Fig. 2(b) above, a slight reduction of the vibrational dressing (RVD) in the LPP is noticed, close to the threshold at which reorganization energy and Rabi frequency become comparable (i.e., when $\Omega_{R} \simeq \Delta$ ).

In particular, numerical and variational solutions for larger $N$ display scaling of this phenomenon as $1 / N[16,17,21]$. In the thermodynamic limit, therefore, the LPP becomes closer to the bare LP, and polaron formation is suppressed. A key observation is that RVD is not observed for the whole ladder of eigenstates, resulting in strong vibronic effects arising in the excited states (for example, in the dynamics triggered by a high-frequency pulsed excitation). One direct consequence of polaron decoupling is the eventual suppression of the reorganization energy of excited electrons, which, as first pointed out by Herrera and Spano in [16], could be exploited to enhance electron transfer reaction rates.

\section{EXCITONIC AND PHOTONIC FEATURES OF THE LPP}

The spectral density in organic materials depends strongly on the molecules and the host matrix. In order to obtain general conclusions, we thus first study the effect of many-mode vibrational dressing on polaritons using a standard parametrization of low-frequency vibrational modes, the Leggett-type spectral density ${ }^{1}$

$$
J_{v}(\omega)=2 \pi \alpha \omega_{c}^{1-s} \omega^{s} \theta\left(\omega_{c}-\omega\right), \quad s>0
$$

where $\omega_{c}$ corresponds to a cutoff energy, $\alpha$ describes the overall strength of the exciton-phonon coupling, and the exponent $s$ determines the shape of the spectral density, with $s=1$ giving a so-called Ohmic spectral density, while $s<1$

\footnotetext{
${ }^{1}$ Spectral densities of this type have been studied thoroughly in the simplest example of quantum dissipation model, the spin-boson model. See [48] and [46] for further details.
} 

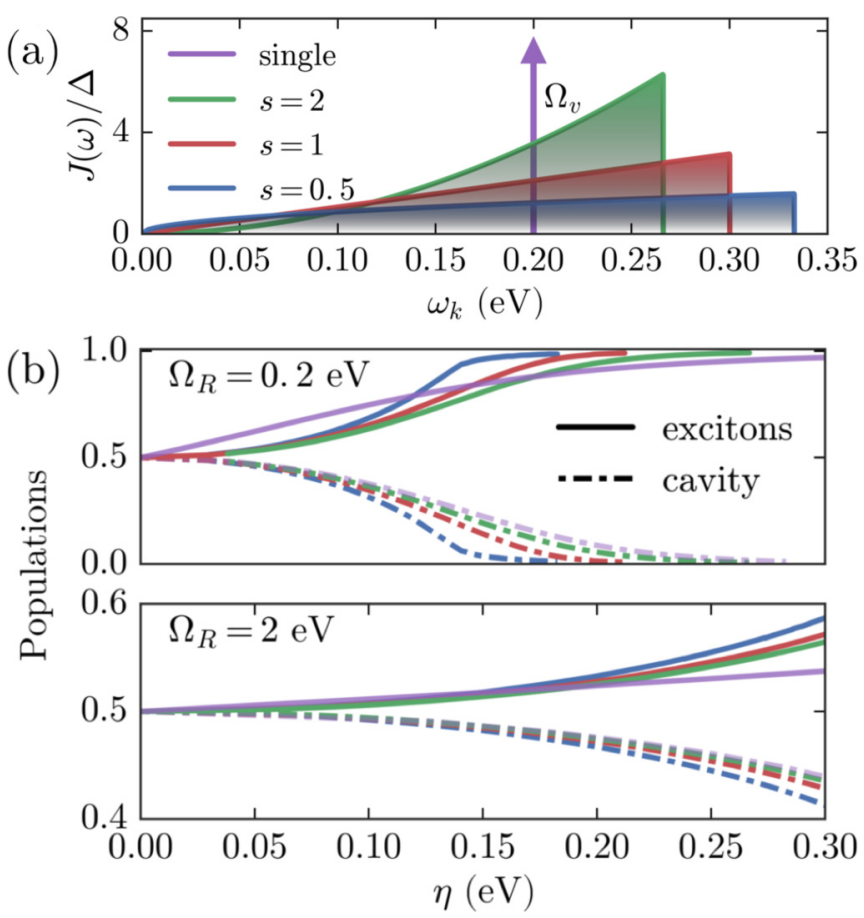

FIG. 4. (a) Functional forms adopted for the LPP calculation and (b) reduced populations over subsystem $\mathcal{S}$ in the LPP as a function of the coupling strength to the reaction coordinate $\eta$. Here, different curves correspond to the different spectral densities in (a), depicting in the upper and lower windows of (b) the cases for Rabi frequency shown.

and $s>1$ correspond to sub- or super-Ohmic densities, respectively. The cutoff $\omega_{c}$ implies that fast modes $\omega_{k}>\omega_{c}$ are reabsorbed in the coupling constants through the adiabatic approximation [46]. It is interesting to note for the following discussion that, within the single-molecule limit, a regime recently reported in plasmonic nanocavities [10,47], the Hamiltonian (2) can be mapped precisely to the wellknown spin-boson model (SBM) [46,48] by a shift of the vibrational mode origin, as shown in Appendix B.

In this section, we study the influence of vibrational dressing on the excitonic and photonic properties of the LPP. We focus on Leggett-type spectral densities [Eq. (7)] with $s=$ $0.5,1$, and 2 [shown in Fig. 4(a)]. For ease of reference, we will compare the results with those obtained from the HTC model. The cutoff frequency $\omega_{c}$ is tuned to maintain the reaction coordinate frequency constant and equal to that of the reference HTC model $\Omega_{v}=\omega_{c}(1+s) /(2+s)\left(=\omega_{v}\right.$ in the HTC). We consider an anthracenelike molecule having a vibrational spacing of $\Omega_{v}=0.2 \mathrm{eV}$, and unless otherwise stated, we assume an ensemble containing $N=5$ molecules.

In order to investigate the interplay between excitonphoton and exciton-phonon interactions without dealing with the full many-body state, we trace out the vibrational modes to calculate the reduced density matrix $\hat{\rho}_{\mathcal{S}}=\operatorname{Tr}_{\mathcal{E}_{v}}\left\{\left|\psi_{-}\right\rangle\left\langle\psi_{-}\right|\right\}$. As Fig. 4(b) shows, the LPP state becomes more excitonic as the coupling amplitude to the reaction coordinate $\eta$ [Eq. (4)] grows, with a more substantial photonic character in a given molecule if the Rabi frequency $\Omega_{R}$ is larger. Such behavior signals the tradeoff between vibrational and photonic coupling scales. Nevertheless, the different curves for different $J_{v}(\omega)$ clearly indicate that the vibrational influence on the system is not solely determined by the reaction coordinate in the organic molecules. Instead, the remaining "dark" vibrational combinations produce changes that are not captured adequately by $\eta$.

These results motivate us to consider the reorganization energy $\Delta$, defined by Eq. (6) and given by $\Delta=2 \alpha \omega_{c} / s$ for Leggett-type $J_{v}(\omega)$, as a measure of the global influence of the whole set of phononic modes on the system. We next proceed to include the LPP eigenfrequency $\omega_{\psi_{-}}$in the discussion. In particular, the values of the shift $\omega_{\psi_{-}-} \omega_{-}$ (the bare LP frequency to disregard the linear energy shift by the Rabi frequency in the discussion) as a function of the reorganization energy can be read from Fig. 5(a). The LPP eigenenergy undergoes a monotonic red-shift as a result of the increased vibrational dressing in electronic and photonic components. Mirroring the results for the HTC model, the slope of the curves differs at either side of the crossover at $\Delta \simeq \Omega_{R} / 2$, being steeper at the large $\Delta$ region [21]. Accompanying this trend, the total photonic (excitonic) fractions of the LPP (diagonal elements in $\hat{\rho}_{\mathcal{S}}$ ) are decreasing (increasing) very similar functions of the reorganization energy [Fig. 5(b)]. Additional insight into the LPP internal structure is gained by considering the weights of the vibration-free eigenstates $(\Delta=0)$ that are mixed into the LPP by the vibronic coupling. As expected, Fig. 5(c) reveals that $\left|\psi_{-}\right\rangle$is constituted by major contributions from the bare LP $(|-\rangle)$ and dark states $(|d\rangle \in \mathcal{D})$, with some small fraction of the UP $(|+\rangle)$. In particular, in the flatter region where $\Delta \ll \Omega_{R} / 2$ the LPP is well approximated by the bare lower polariton with frequency $\omega_{\psi_{-}} \simeq \omega_{-}=\omega_{e}-\Omega_{R} / 2$

The results above suggest that in real space the limit at large Rabi frequencies corresponds to a LPP that is a spatially delocalized state over the ensemble, with nearly no molecular phonon excitation. In the opposite large Stokes-shift limit, the LPP becomes closer to spatially localized polarons, with a LPP-LP shift of $-\Delta$ [irrespective of $J_{v}(\omega)$ ], and no photonic component. The tradeoff between the two effects leads to a relocation of the contribution $\rho_{--}$, into the dark excitonic states $\rho_{\mathcal{D}, \mathcal{D}}$, with eventual crossover at the large Stokes-shift limit, mirroring results for the HTC model in the previous section. To further corroborate the existence of the previous extremes we calculate the bipartite entanglement between $\mathcal{S}$ and $\mathcal{E}$, measured by the partial von Neumann entropy $S\left(\hat{\rho}_{\mathcal{S}}\right)=$ $-\operatorname{Tr}\left(\hat{\rho}_{\mathcal{S}} \log \hat{\rho}_{\mathcal{S}}\right)$. As we observe in Fig. 5(d), correlations in the LPP, absent at $\Delta=0$, build up for increasing Stokes shift as vibrational states become more mixed with excitons (and indirectly the cavity photon). For large $\Delta$, the formation of polarons entails the saturation of the entanglement entropy at the value $S\left(\hat{\rho}_{\mathcal{S}}\right) \simeq \log N$ corresponding to $N$ excitons maximally mixed with the vibrational modes.

In the halfway of the polaritonic and polaronic limits, where neither $\Omega_{R}$ nor $J_{v}(\omega)$ are negligible, state $\left|\psi_{-}\right\rangle$ possesses a threefold excitonic-photonic-polaronic character. Even in this region, observables acting within the system $\mathcal{S}$, present only quantitative changes depending on the vibrational spectrum of the molecules. A direct consequence is the emergence of an RVD effect when comparing the LPP with uncoupled excitons, arising from an increased Rabi 

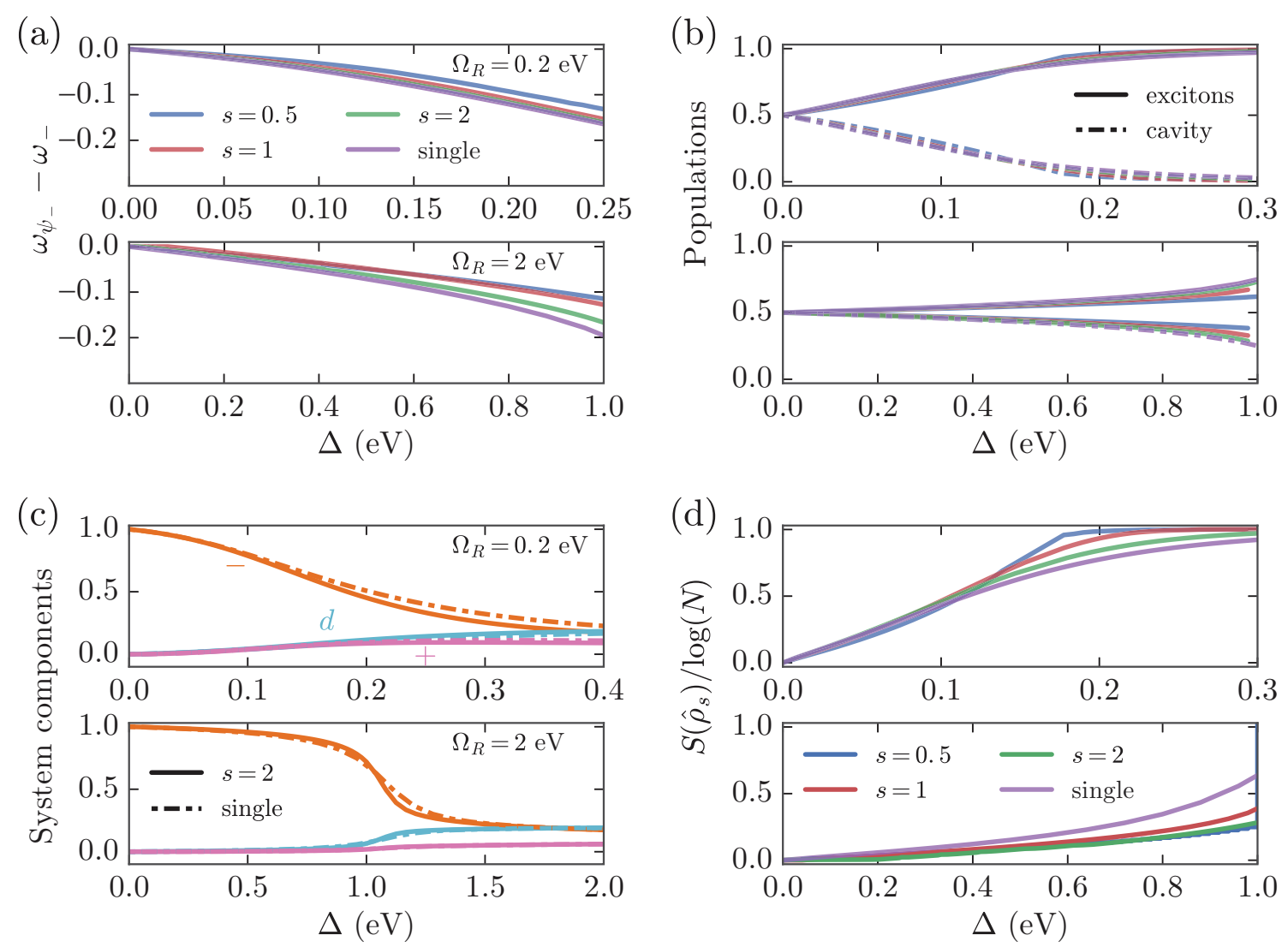

FIG. 5. (a) Energy shift with respect to the bare LP ( $\Delta=0$ ), (b) exciton/photon fractions (c), vibration-free components in the LPP, and (d) von Neumann entropy of the photonic-excitonic and vibrational states bipartition, as a function of reorganization energy. In these panels, upper panels depict the case $\Omega_{R}=\Omega_{v}$ and $\Omega_{R}=10 \Omega_{v}=2 \mathrm{eV}$ for the lower panels.

frequency (lower panels in Fig. 5). In this way, shallower curves in Figs. 5(a)-5(c) indicate a larger resemblance with the LP in frequency, populations, and light-matter coherences, respectively, while the entanglement entropy $S\left(\hat{\rho}_{\mathcal{S}}\right)$ diminishes in Fig. $5(\mathrm{~d})$ as $\Omega_{R}$ is augmented due to decoupling from vibrations. In our simulations, we also observed similar effects for a fixed Rabi frequency and growing ensemble size $N$. The increasing suppression of the vibrational dressing could suggest the onset of polaron decoupling in the limit $N \gg 1$, similar to the one reported for a single mode as observed above [16,21]. From the point of view of chemistry, similar results have been obtained without canonical quantization of molecular vibrations, generalizing our conclusions to arbitrary electronic potential energy surfaces far from equilibrium (e.g., in chemical reactions) [17,49]. In this case, the so-called "collective protection" (the extended version of the RVD) has been shown in the thermodynamic limit, by means of suppressed displacements in the polaritonic (nuclear-coordinateresolved) surfaces. Lower-polariton and ground-state (vacuum) electronic surfaces thus become identical, with a critical impact on photoisomerization reactions.

The results above reveal the robustness of the RVD, which does not rely on the fine molecular/host details encoded in $J_{v}(\omega)$. Instead, the LPP for molecules close to the equilibrium is accurately characterized by the coupling to a reaction coordinate with frequency $\Omega_{v}$ while the global effect of the vibrational modes enters through the full reorganization energy $\Delta$.
These features can therefore be understood within an effective HTC model, with phonon frequency chosen according to the experimental reaction coordinate frequency and Stokes shift.

The case of polaritons formed from molecules with large Stokes shift has been recently reported, showing that emission from the hybrid polaritons is suppressed and instead occurs from the bare molecules [50]. In this limit, it is more energetically favorable for an exciton to form a polaron rather than hybridize with a photon, as shown by diagonalization of $\hat{H}_{\mathcal{S}}+\hat{H}_{v}$. Although TN based approaches to target excited states have been devised [51], we exploit aforementioned similarities to further visualize the photonic properties of a simpler HTC model with $N=2$ (see Fig. 6). In this manner, we observe that the quasiparticle weight of the vibrationfree polariton $\mathcal{Z}_{\psi_{n}}=\left\|\left\langle\psi_{-} \mid \psi_{n}\right\rangle\right\|^{2}$ indeed "climbs up" to the excited states as $\Delta$ is increased, a fingerprint of polaritons at higher energies (see Fig. 6). This can be understood as a consequence of the fact that transitions within the FranckCondon region (and thus with non-negligible dipole moment) occur to vibrationally excited states for large exciton-phonon coupling.

Finally, we noticed quantitative differences as molecular details were varied, which are particularly prominent in the region $\Delta \simeq \Omega_{R} / 2$, where maximum mixing between purely polaritonic and polaronic states is observed, and when the vibrational spectrum in the molecules is dominated by slow modes [sub-Ohmic $J_{v}(\omega)$ ]. While exciton/photon properties have been shown to be universal, the impact of light-matter 


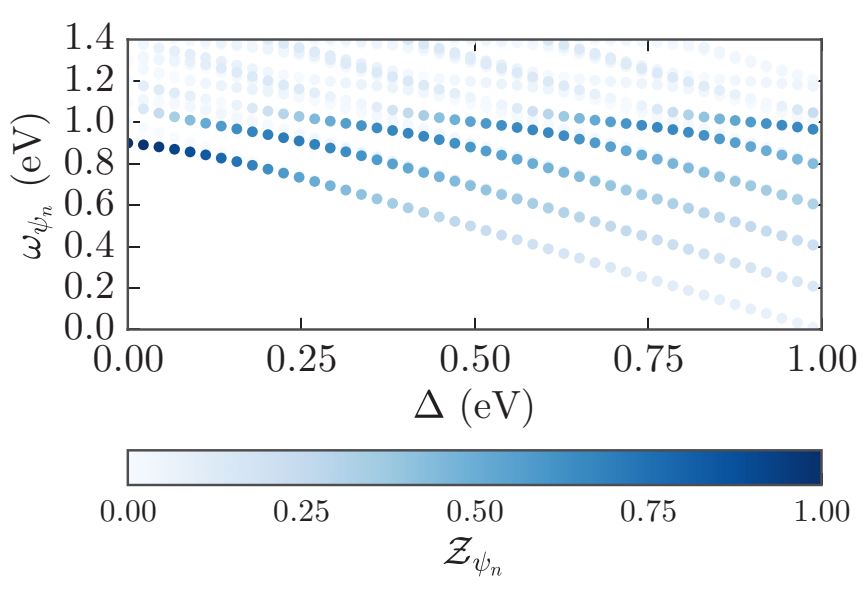

FIG. 6. Excitation spectrum $\left|\psi_{n}\right\rangle$ of $\hat{H}_{\mathcal{S}}+\hat{H}_{v}$ for a HTC model with $N=2$ molecules and $\Omega_{R}=0.2 \mathrm{eV}$. The color scale indicates the overlap with the bare LP $\mathcal{Z}_{\psi_{n}}$ with increasing saturation.

coupling on different molecular vibrations is averaged out in $\hat{\rho}_{\mathcal{S}}$. These nontrivial features motivate further analysis of the intrinsic vibrational properties in the LPP state, by first calculating a quasiparticle weight $\mathcal{Z}_{\psi_{-}}=\left\|\left\langle\psi_{-} \mid-\right\rangle\right\|^{2}$ that indicates how close the LPP is to the vibration-free LP, shown in Fig. 7. Increased overlaps signal the RVD at large Rabi frequencies and exponents $s$. It should be noted that $\mathcal{Z}_{\psi_{-}}$ includes only the overlap with the vibrational vacua $|0\rangle_{\mathcal{E}_{v}^{(i)}}$, in contrast with the population $\rho_{--}$, reduced over the whole environment. Therefore, when Figs. 5(c) and Fig. 7 are compared, a more polaronic character of the LPP is noticed with larger vibrational dressing for sub-Ohmic $J_{v}(\omega)$. However, as we show in Fig. 5(a), the energy shifts associated with sub-Ohmic baths are actually weaker than in super-Ohmic and single-mode environments, indicating that the RVD is strongest for sub-Ohmic baths. We shall elucidate the origin of this effect, related to the dominance of slow vibrational modes in sub-Ohmic environments, in the next section by further exploring the many-body vibrational properties of the LPP state.

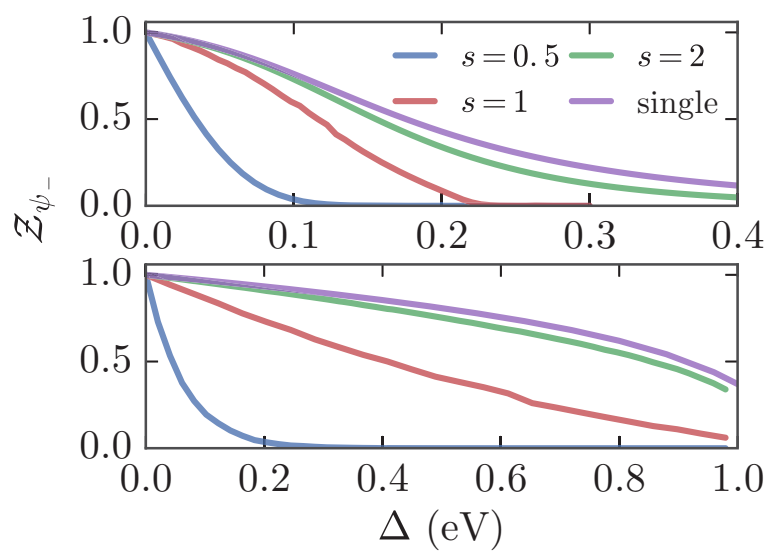

FIG. 7. Overlap of the LPP wave function with the vibration-free polariton $|-\rangle$ for $\Omega_{R}=0.2 \mathrm{eV}$ (upper panel) and $\Omega_{R}=2 \mathrm{eV}$ (lower panel) for the HTC and $J_{v}(\omega)$ in the form (7).

\section{VIBRATIONAL FEATURES OF THE LPP}

Our VMPS approach enables access to the full many-body vibrational component of the LPP wave function, which can be exploited to resolve phononic features in frequency space that are disregarded in the HTC model. In this section we show how vibrational observables are shaped by the specific shape of $J_{v}(\omega)$. To this end, we analyze the frequency-resolved vibrational displacement in the LPP state for the electronically excited molecule $i$. For further insight we split this observable into conditional displacement contributions arising from each system state $\left|n_{\mathcal{S}}\right\rangle \in \mathcal{S}$ mixed into the LPP,

$$
\mathcal{X}_{i, n_{\mathcal{S}}}\left(\omega_{k}, \Omega_{R}, \Delta\right)=\frac{\left|\left\langle\psi_{-}\left|\hat{\mathcal{P}}_{n_{\mathcal{S}}}\left(\hat{b}_{k}^{(i)}+\hat{b}_{k}^{(i) \dagger}\right)\right| \psi_{-}\right\rangle\right|}{\rho_{n_{\mathcal{S}}, n_{\mathcal{S}}}},
$$

which includes a projector over system states $\hat{\mathcal{P}}_{n_{\mathcal{S}}}$. Here, we normalized by the corresponding system-state population to discern situations where the state fraction is vanishingly small. In practice, the calculation of Eq. (8) requires reverting the chain mapping for the $\hat{c}_{l}^{(i)}$ modes.

The displacement spectra in Fig. 8 explicitly show the RVD of electronically excited molecules in the LPP, with decreased vibrational displacement $\mathcal{X}_{i, e_{i}}$ from the bare-molecule value $\mathcal{X}_{i, e_{i}}\left(\omega_{k}, \Omega_{R}=0\right)=\lambda_{k}^{2} / \omega_{k}^{2}$, when $\Omega_{R}$ is increased [see Fig. 8(a)] and a more substantial suppression at low frequencies and smaller $s$.

The microcavity mode induces a "crosstalk" between otherwise disconnected molecules (note that we neglected dipole-dipole interactions). This results in a finite contribution of phononic displacement in a given molecule, caused by electronically excited states residing in others, $\mathcal{X}_{i, e_{j \neq i}}$ [see Fig. 8(b)], and also a "molecule-induced" vibrational displacement in the cavity state measured by $\mathcal{X}_{i, 1}$. Although these contributions are suppressed for low frequencies at large $\Omega_{R}$, in a similar way to the "local" quantity $\mathcal{X}_{i, e_{i}}$, they slightly augment in a counterintuitive way for larger frequencies. Nonlocal and cavity-mode vibrational dressing present very similar trends, while the second is less sensitive to the Rabi frequency $\Omega_{R}$ [see Fig. 8(c)]. Moreover, an analysis varying the ensemble size at large $\Omega_{R}$ for $N=1-5$ (not shown) suggests the local vibrational displacement $\mathcal{X}_{i, e_{i}}$ scales for the whole frequency spectrum as $1 / N$. These results generalize the scaling predicted by the variational Ansatz solution for the HTC [21], which is related with the prefactor $1 / \sqrt{N}$ in the coupling between bare electronic states in the LPP. Nonlocal and cavity-projected vibrational dressing also appear to follow a universal trend of $1 / N$ in the cases analyzed here. Therefore, despite the indirect connection of molecular vibrations through the cavity in strong coupling, vibrational dressing is collectively suppressed. At moderate Rabi frequency, the situation is different, and the reduction with $N$ depends nonanalytically on $\Omega_{R}$ and $\Delta$. Although a full analysis is beyond the scope of this contribution, we note that the related SBM possesses a set of quantum phase transitions for Ohmic and sub-Ohmic baths in which strong polaronic dressing completely suppresses tunneling dynamics. For sub-Ohmic baths, the critical coupling is a function of quantities related to $\Omega_{R}$ and $\Delta$ (see Appendix B) $[48,52]$, and the ground state contains complex superpositions of displaced oscillator states 

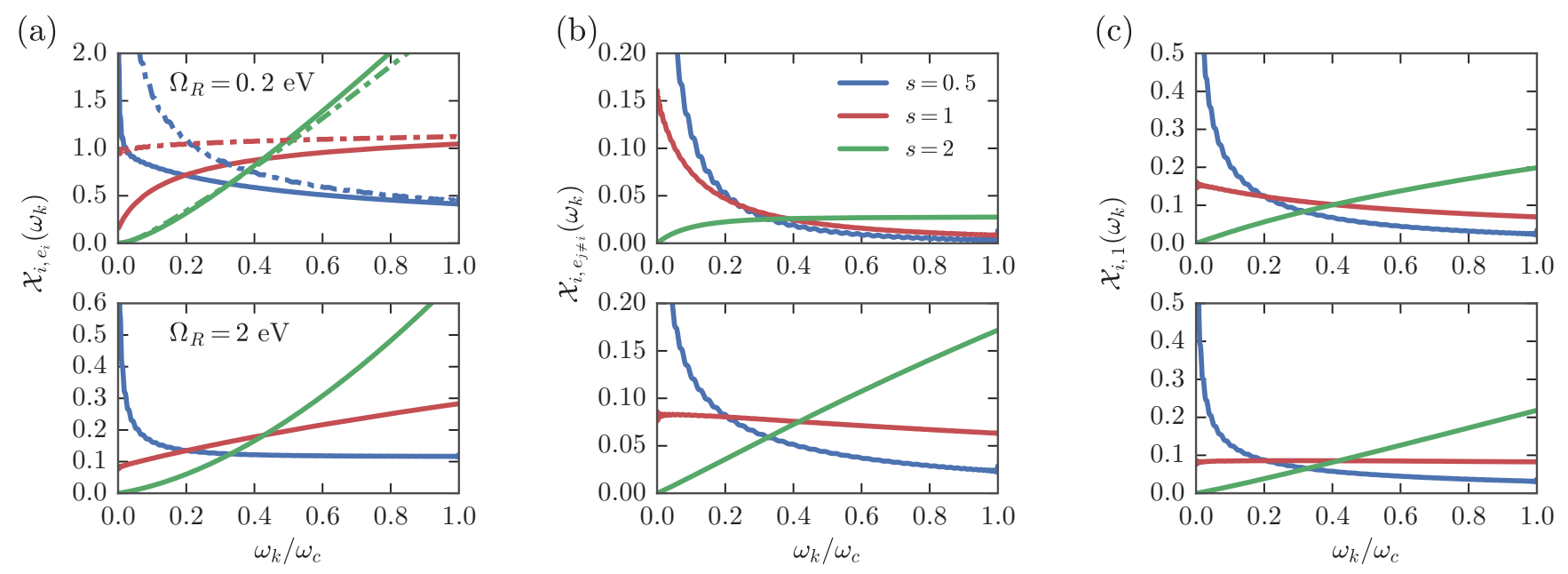

FIG. 8. Absolute displacement spectrum of the phononic modes for a molecule $i$, projected on (a) the electronic state of the excited molecule, the electronic state of the unexcited molecule (b), and the cavity photon (c). In these panels, we considered $\Omega_{v}=0.2 \mathrm{eV}, \Delta=0.1 \mathrm{eV}$, and $N=5$. The limit $\Omega_{R} \rightarrow 0$ is displayed in dashed-dotted lines.

[52], which may be relevant for the physics of intermediate Rabi couplings.

As for the bare molecules, this observable is extremely sensitive to the molecular species supporting the LPP, even when the Stokes shift is kept constant. Additional insight into the strength of the RVD effect for a given molecular ensemble is given by the ratio

$$
\mathcal{R}\left(\omega_{k}\right)=\frac{\mathcal{X}_{i, e_{i}}\left(\omega_{k}, \Omega_{R}^{\prime}, \Delta\right)}{\mathcal{X}_{i, e_{i}}\left(\omega_{k}, \Omega_{R}, \Delta\right)},
$$

where $\Omega_{R}^{\prime}>\Omega_{R}$ are two Rabi frequencies. Intriguingly, as shown in Fig. 9, the ratio $\mathcal{R}$ is mostly independent of the power law in $J_{v}(\omega)$, unveiling another universal characteristic of the model. Therefore, although the LPP is more polaronic for lower $s$, the suppression at low frequencies is increased in absolute terms in such a way that the relative suppression spectrum is molecule independent.
Exploiting the similarities with the SBM for a single molecule, as discussed in Appendix B, the intrinsic frequency dependence of $\mathcal{R}$ follows from the variational polaron theory for the SBM $(N=1)$ with "bias" $\delta=-\Delta$ (provided that $\left.\omega_{e}=\omega_{O}\right)$. For large $\Omega_{R}$ and small $\Delta$, bias effects caused by the vibrational reorganization energy become irrelevant. The slow modes are antiadiabatic from the perspective of the Rabi oscillations. Hence, they cannot readjust their displacements rapidly enough to track the formation of polaritons arising from light-matter coupling [32], and are unable to maximize the vibrational dressing of the electronic states. Vibrational and photonic processes are hence "decoupled" and $\mathcal{R}$ decreases monotonically with the frequency. This picture agrees with the smaller energy shifts for Ohmic and sub-Ohmic spectral densities, corresponding to "slow baths" [see red and blue curves in Figs. 8(a) and 4(a)]. Conversely, larger exponents $s$ correspond to spectral densities where high-frequency modes are dominant. In particular, these modes are adiabatic from the
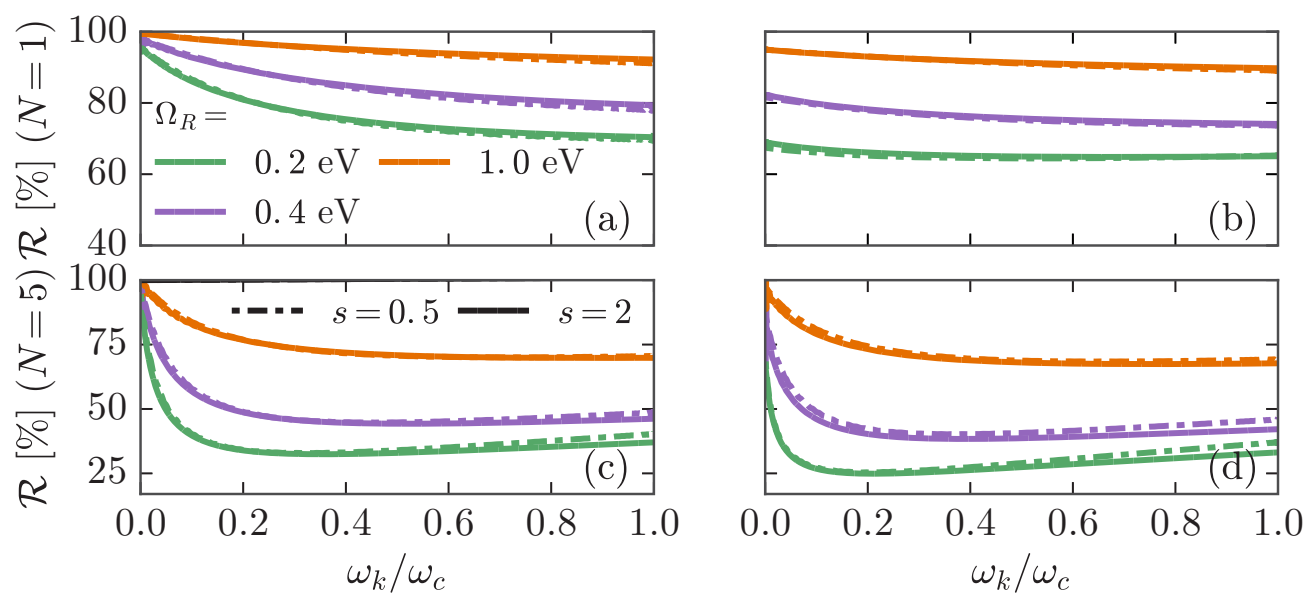

FIG. 9. Ratio $\mathcal{R}$ between displacement at $\Omega_{R}^{\prime}=2 \mathrm{eV}$ and lower Rabi frequency displacements, for the $\mathrm{SBM}$ at $N=1$ (a), (b), and $N=5$ (c), (d). The case $\Delta=0.01 \mathrm{eV}$ is shown in left panel [(a), (c)] while $\Delta=0.1 \mathrm{eV}$ is depicted in the right [(b), (d)]. Two distant power laws $s=0.5$ and 2 are shown. 

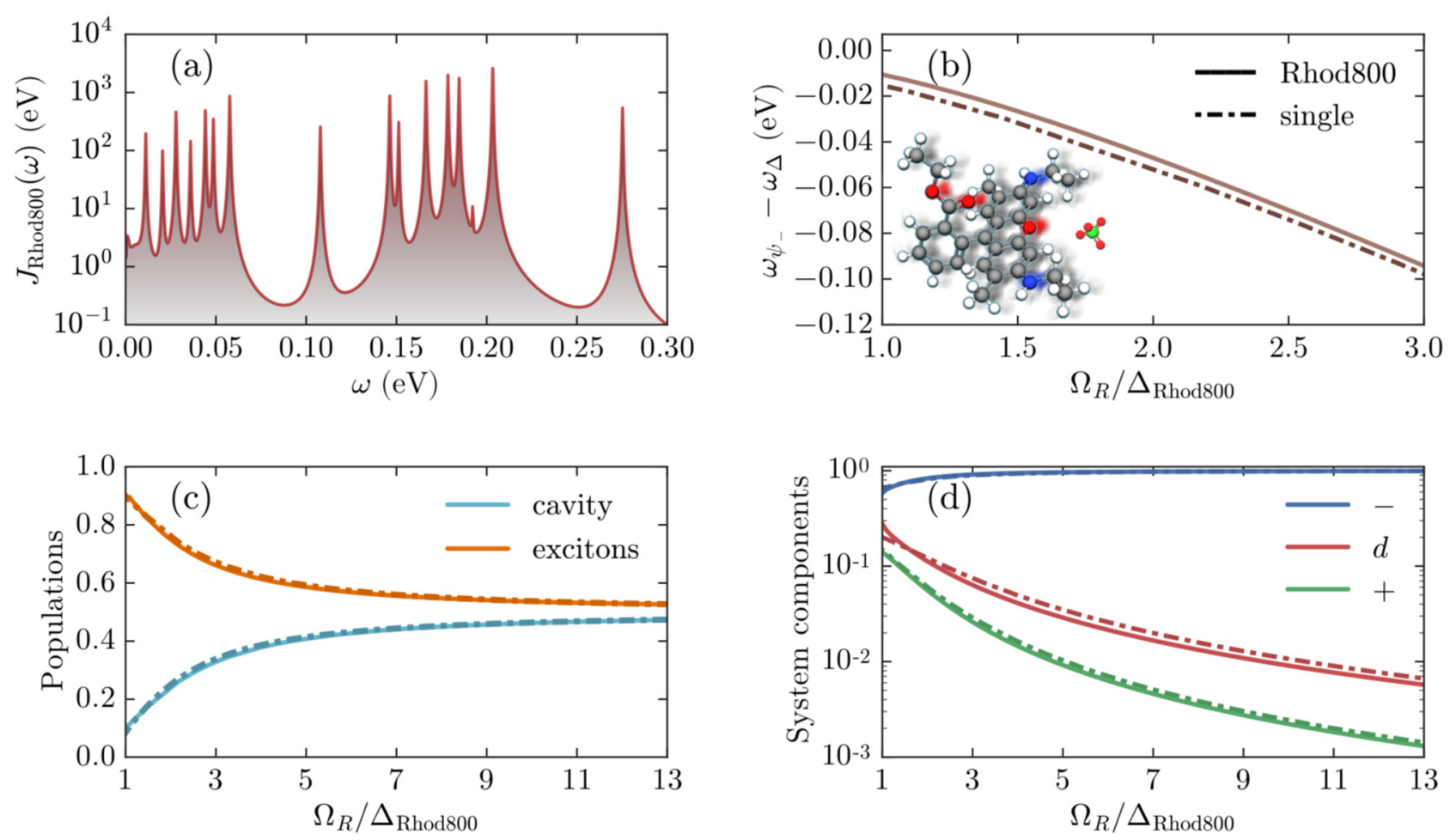

FIG. 10. Reduced observables for the LPP supported by a dimer of Rhod800 molecules. The total reorganization energy for the modes that are considered is $\Delta=0.112 \mathrm{eV}$ (here $\omega_{\Delta}=\omega-\Delta$ ). The numerical first moment of the spectral density $J_{\text {Rhod800 }}(\omega)$, depicted in (a), reveals a value of $\Omega_{v}=0.154 \mathrm{eV}$.

$\mathcal{S}$ subsystem perspective and lead to larger frequency shifts and phononic displacements. Besides, such effects could induce renormalization of the coupling parameters, which could be observed for instance in a reduced Rabi splitting in linear response spectrum.

The results for $N=5$ molecules displayed in Fig. 9 (lower panels) show a more significant suppression of vibrational displacement compared with the SBM. In this case, RVD occurs by the additive contribution of the light-matter coupling, described above, and ensemble effects such as the "collective protection" due to each molecule in the polariton state staying mostly in its ground state [17]. Intriguingly, a global minimum in $\mathcal{R}$ is observed for a finite $\omega_{k} \sim \mathcal{O}\left(\Omega_{R}\right)$, suggesting nontrivial bias effects due to non-negligible reorganization energy $\Delta$ [52]. Indeed, it has recently been shown that the very large displacements associated with the strongly coupled slow modes in the sub-Ohmic spin-boson model may develop over very long timescales when the system is biased and then prepared out of equilibrium, i.e., by laser excitation [53,54]. This creates an effective time-dependent bias which, as our static results suggest, could dynamically alter polaritonic properties in real time and generate strongly non-Markovian dynamics that might be detectable in timeresolved microcavity experiments.

The results outlined in this section clearly suggest that the HTC model is insufficient to address the nontrivial vibrational features arising for different molecular systems $\left[J_{v}(\omega)\right]$ in the LPP, but that instead it is necessary to take into account the vibrational structure of the molecule. Nevertheless, we have shown that the relative effect of RVD is somewhat irrespective of the spectral density of molecular phonons but is mostly determined by the reaction coordinate frequency and the reorganization energy.

Up to now, we have considered smooth vibrational spectral densities. In the following, we show how these ideas can be tested in a molecule with highly structured vibrational features that recreates the conditions found in realistic organic microcavities.

\section{RHODAMINE 800 MOLECULE}

In typical organic molecules, aside from simple power-law spectra at low frequencies, more complicated features may arise at high (typically mid-infrared) frequencies, as observed in, e.g., infrared and Raman spectroscopy where many sharp vibrational resonances are present. In the following, we consider the rhodamine 800 perchlorate molecular compound [sketched in Fig. 10(b)], which constitutes a common choice for a laser gain media and strong coupling experiments [55]. In this case, the phononic information necessary to "feed" $J_{v}(\omega)$ is known from vibrational spectroscopy [56]. In particular, the data from three-pulse photon echo peak shift (3PEPS) experiments at room temperature (in Fourier space) can be interpolated to give an accurate approximation for the low-frequency part $J_{v}^{\text {lf }}(\omega)$ [57]. Regarding the high-frequency modes, denoted as $\Omega_{k}$, we consider that each oscillator is broadened by an amount of $\Gamma \ll \Omega_{k}$ due to the interaction with a background Ohmic bath of vibrations accounting for 

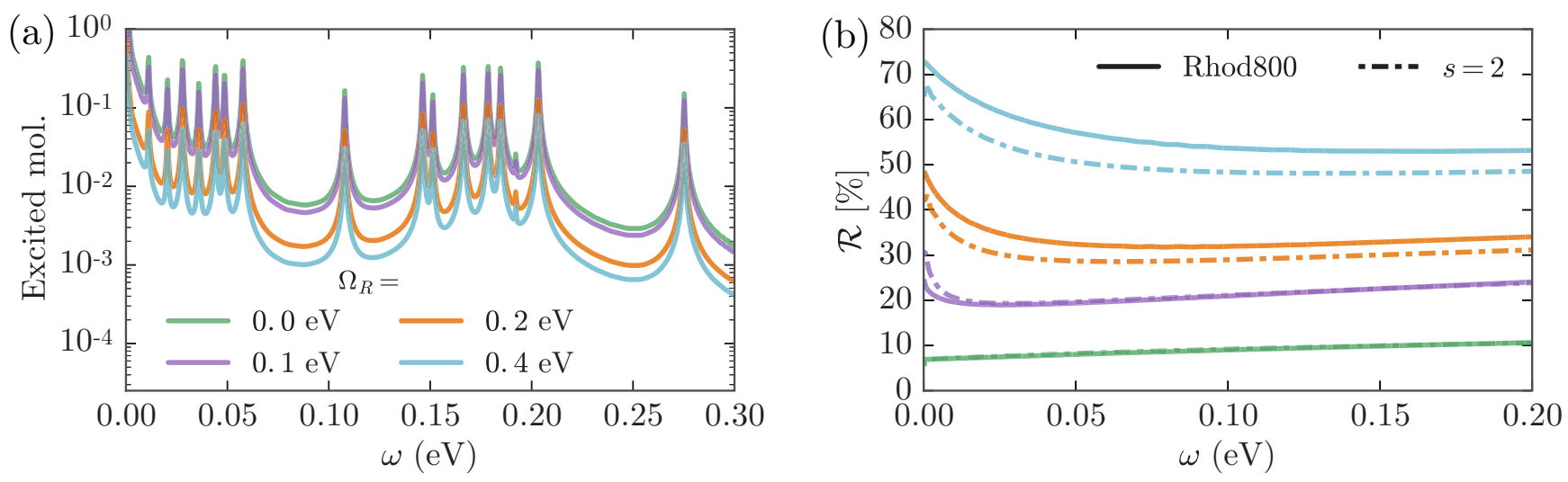

FIG. 11. (a) Displacement spectrum for the LPP supported by a dimer of Rhod800 molecules, projected on the electronically excited molecule. (b) Ratio between the displacement of the electronically excited molecule at $\Omega_{R}^{\prime}=1.5 \mathrm{eV}$ and the cases depicted in (a). The remaining parameters are fixed according to Fig. 10 caption.

vibrational dephasing and decay [33], by taking

$$
J_{v}^{\mathrm{hf}}\left(\omega, \Omega_{k}, f_{k}, \Gamma\right)=\frac{f_{k} \omega}{\left(\Omega_{k}^{2}-\omega^{2}\right)^{2}+(\Gamma \omega)^{2}},
$$

where $\Gamma$ measures the mode broadening and $f_{k}$ is proportional to vibronic coupling to mode $k$. First, we compared the $\mathcal{S}$ populations of a dimer of Rhod800, with spectral density $J_{\text {Rhod800 }}(\omega)=J_{v}^{\text {lf }}(\omega)+\sum_{k \in \text { hf }} J_{v}^{\text {hf }}\left(\omega, \Omega_{k}, f_{k}, \Gamma\right)$, shown in Fig. 10(a), with a HTC model with parameters $\Omega_{v}^{\mathrm{Rhod} 800}, \Delta^{\mathrm{Rhod} 800}$ estimated from the experimental spectral density. Strikingly, despite the involved resonant structure of $J_{\text {Rhod800 }}(\omega)$, the energy shift and the reduced density matrix observables present a smooth behavior as the Rabi frequency $\Omega_{R}$ is varied (Fig. 10), which mimics the HTC model. This predicts that the probing of excitonic and photonic characteristics of the LPP supported by an ensemble of arbitrarily complex molecules, close to the mechanical equilibrium, only requires the experimental knowledge of few collective quantities.

However, in agreement with the conclusions drawn for the polynomial spectral densities in Eq. (7), the vibrational features cannot be described by the HTC model but are profoundly dominated by the vibrational structure of the molecule and the host medium, encoded in $J_{v}(\omega)$. Phononic displacements of the exciton component of the LPP in Fig. 11(a) mirror the resonant structures in $J_{\text {Rhod800 }}(\omega)$. Nevertheless, the relative suppression $\mathcal{R}$ is surprisingly smooth [see Fig. 11(b)]. In agreement with our previous results, it is controlled by just the reaction coordinate frequency $\Omega_{v}$ and the reorganization energy $\Delta$ instead of details in $J_{v}(\omega)$.

\section{CONCLUSION}

In this work, we have investigated a microscopic theory that goes beyond current models for the lower polaronpolariton in organic microcavities by introducing the whole phononic spectrum. In order to treat the problem numerically, we employed a quasiexact VMPS algorithm, able to handle the full Hamiltonian including multimode and many molecule effects. We have demonstrated that the RVD of polaritons compared to bare electronic states is a universal feature of strongly coupled organic microcavities that does not depend on the details of the vibrational structure. In this way, different distributions of intramolecular or extramolecular vibrational modes lead to similar excitonic and photonic properties of the LPP wave function, where only the bath component is appreciably altered.

Our findings show unambiguously how, despite the involved phononic spectrum in organic molecules, a large extent of features in the LPP can still be emulated by the HTC model. The universal character of the results is not expected to be present in the time evolution of the exciton-photonic system, a problem that has attracted interest lately due to the experimental advances achieving exotic organic polariton dynamics [58] and nonequilibrium BEC in the strong coupling regime [59]. As we show in [30], the nonequilibrium description of the bath modes is crucial to determine the time-evolution route followed by the system arising from details in $J_{v}(\omega)$ [60].

However, there is still a long way to go regarding experiments to obtain conclusive evidence of changes in chemical processes or electronic energy transfer in electronic strong coupling. The model presented and explored here seeks to generalize the most simple descriptions of the problem, while future prospects along these lines would go towards extended approaches, that are able to introduce multidimensional features in the recent field of polaritonic chemistry.

\section{ACKNOWLEDGMENTS}

This work has been funded by the European Research Council (Grants No. ERC-2011-AdG-290981 and No. ERC-2016-STG-714870), and the Spanish MINECO under Contract No. MAT2014-53432-C5-5-R and the "María de Maeztu" programme for Units of Excellence in R\&D (MDM2014-0377). F.A.Y.N.S. and A.W.C. gratefully acknowledge the support of the Winton Programme for the Physics of Sustainability and EPSRC.

\section{APPENDIX A: TENSOR NETWORK BASED APPROACH}

In this appendix, we outline the numerical approach to target the optimal LPP $\left|\psi_{-}\right\rangle$. We state only what is necessary to understand this work, referring to the reader to complete reviews to the topic $[26,61]$. 
(a)

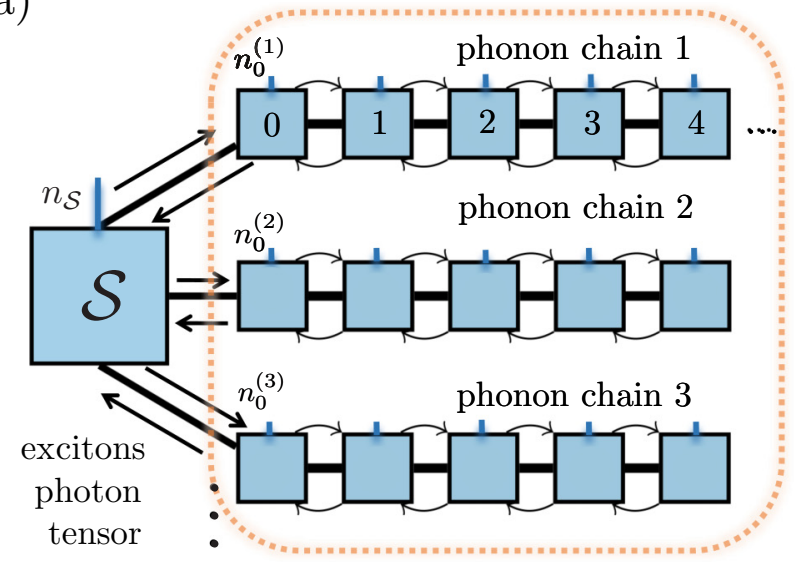

(b)

(b) MPS but site $l$

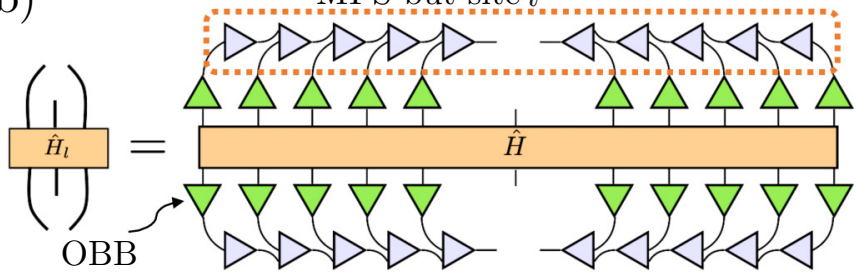

isometry

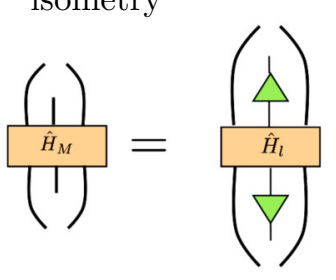

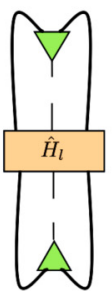

FIG. 12. (a) Sketch of the star TN for the case $N=3, M_{v}=5$ that is used to represent the LPP. Here, each of the squares signifies a tensor, that is contracted with the tensor to which its legs are connected. Arrows depict a possible order at which the tensors are optimized in the algorithm. The open legs represent the local Hilbert space at each of the sites, which is contracted with the Hamiltonian $\hat{H}=\hat{H}_{\mathcal{S}}+\hat{H}_{v}$ to state the minimization problem. To carry out this minimization, it is instrumental to represent the state in mixed canonical form compute the contractions depicted in (b). Here, we use a similar notation for the tensors as in the [63].

The numerical approach is based on the two following ideas: (i) Representation of $\left|\psi_{-}\right\rangle$as a multichain TN, mimicking the structure in the star Hamiltonian Fig. 1 that can be employed to implement the (ii) variational principle

$$
\left|\psi_{-}\right\rangle=\min _{|\psi\rangle \in \mathcal{M}_{\mathrm{TN}}} \frac{\left\langle\psi\left|\hat{H}_{\mathcal{S}}+\hat{H}_{v}\right| \psi\right\rangle}{\langle\psi \mid \psi\rangle},
$$

within the TN submanifold of the total Hilbert space $\left(\mathcal{M}_{\mathrm{TN}} \subset\right.$ $\mathcal{H}_{\mathcal{S}} \otimes \mathcal{H}_{\mathcal{E}_{v}}$ ) that leads to the star-DMRG algorithm employed in the work.

\section{Star TN structure}

The DMRG algorithm works especially well targeting ground states in one-dimensional systems. The orthogonal mapping of the vibrational modes, leading to the star Hamiltonian introduced in the main text, constitutes a convenient starting point for the application of this numerical method. We consider the expansion of the LPP in the single exciton-photon subspace, which exploits the Fock states $\left|n_{l}^{(i)}\right\rangle \in \mathcal{E}_{v}^{(i)}$ for the $l$ th site at chain $i$, i.e.,

$$
\left|\psi_{-}\right\rangle=\sum_{n_{\mathcal{S}}=1}^{N+1} \sum_{i=1}^{N} \sum_{\boldsymbol{n}^{(i)}} \Psi_{n_{\mathcal{S}}, \boldsymbol{n}^{(i)}}\left|n_{\mathcal{S}}\right\rangle \otimes\left|\boldsymbol{n}^{(i)}\right\rangle,
$$

where the array $\boldsymbol{n}^{(i)}=\left(n_{0}^{(i)}, \ldots, n_{L-2}^{(i)}\right)$ encompasses the Fock numbers at each of the chains. This state admits a representation as a TN. In this case the complex $\left(M_{v}+1\right)$ th-order tensor $\Psi_{n_{\mathcal{S}}, \boldsymbol{n}^{(1)}, \ldots, \boldsymbol{n}^{(N)}}$ is decomposed into a product of rectangular matrices with site-dependent dimensions $d_{l}$ under the following setting: each of the excitations of a given bosonic site and chain $n_{l}^{(i)} \in(0,1, \ldots, \infty)$ is assigned to a matrix $A_{d_{l-1}, d_{l}}^{n_{l}^{(i)}} \in$ $\mathbb{C}^{D_{l-1} \times D_{l}}$, while each of the system degrees of freedom corresponds to $A_{1, d_{1}}^{n_{\mathcal{S}}} \in \mathbb{C}^{1 \times D_{\mathcal{S}}}$. Inherited from the star Hamiltonian depicted Fig. 1, the most natural TN representation of $\left|\psi_{-}\right\rangle$ that enables to reduce the minimization Eq. (A1) to a singlesite problem, in the way described in Appendix A 2, is given by

$$
\left|\psi_{-}\right\rangle=\sum_{n_{\mathcal{S}}=1}^{N+1} \mathbf{A}^{n_{\mathcal{S}}} \sum_{i=1}^{N} \sum_{\boldsymbol{n}_{i}}\left(\mathbf{A}^{n_{0}^{(i)}} \ldots \mathbf{A}^{n_{M_{v}-1}^{(i)}}\right)\left|n_{\mathcal{S}}\right\rangle \otimes\left|\boldsymbol{n}^{(i)}\right\rangle .
$$

The order in which the A matrices occur in the product, sketched in Fig. 12(a), mimics how the sites are connected in the Hamiltonian (3). Instead, here the system tensors (site 0) corresponding to the 0th site are contracted with the boson tensor at each of the 1st sites. To account for bosonic degrees of freedom, a decomposition of the bath modes in terms of an optimal boson basis (OBB) is considered $[43,44]$. The auxiliary indexes that are contracted via the matrix product $d_{l} \in$ $\left(1,2, \ldots, D_{l}\right)$ are known as bond dimensions. They embody the entanglement content of the state; higher bond dimensions signify more entanglement [26], such that for sufficiently high $D_{l}$ the form (A3) can represent any quantum state in the Hilbert space.

The major power of TN based approaches relies on the efficient computer representation of the site tensors $\mathbf{A}$. In practice, this "compression" is achieved by successive iterations of singular value decompositions and truncation of the states with small singular values, corresponding to the Schmidt coefficients [61]. This protocol reduces the maximum bond dimensions, enabling to restrict the possible states to a submanifold $\mathcal{M}_{\mathrm{TN}} \subset \mathcal{H}_{\mathcal{S}} \otimes \mathcal{H}_{\mathcal{E}_{v}}$ that comprises the lowlying many-body states of the system.

\section{Star DMRG algorithm}

Employing the star TN representation (A3) in conjunction with the star Hamiltonian, the minimization problem (A1), limited to the manifold $\mathcal{M}_{\mathrm{TN}}$, can be performed by a 
DMRG-sweeping procedure, which optimizes one matrix at a time while keeping all others fixed, then optimizing the neighboring matrix, and so forth, until convergence is achieved. The basic steps of the algorithm employed, based on sequential 1D DRMG [61] sweeps along the chain, are sketched in Fig. 12(a).

To allow for fast contractions we keep the state in a mixed canonical form, enabling to benefit from orthogonality conditions as shown in [62]. Such representation reduces each DMRG step as a linear eigenvalue (optimization) problem for a single-site tensor (either $\mathbf{A}_{l}$ or the OBB matrices) with the effective Hamiltonians shown in Fig. 12(b). More details about the numerical implementation can be found in [44].

Finally, regarding the convergence of the DMRG algorithm for dense spectra where $M_{v} \gg 1$, it is important to notice that for gapless finite-bandwidth $J_{v}(\omega)$, it has been shown [64] the chain becomes asymptotically homogeneous, such that $\omega_{l \gg 1} \rightarrow \omega_{c} / 2$ and $t_{l \gg 1} \rightarrow \omega_{c} / 4$, respectively. The translational-invariant slice of the chain can be diagonalized in "chain momentum" space, giving rise to a cosine energy dispersion band $\sim \omega_{c}(1-\cos \pi q)$ with a minimum at zero energy. Thus, there is no net contribution of these sites to the LPP energy, and the truncated chain approximation considered in this work is fulfilled.

\section{APPENDIX B: SINGLE-MOLECULE MULTIMODE LIMIT}

One interesting case of the Hamiltonian under study is the single-molecule limit. In particular, single-molecule strong coupling has been recently reported in plasmonic nanocavities $[10,47]$. Within the single electronic/photonic excitation subspace, this case can be mapped exactly to the well-known spin boson model (SBM) $[46,48]$ by a shift of the vibrational mode origin in the original Hamiltonian (1) before performing the chain transformation. After this shift, described by $\hat{H}_{v}^{\text {shift }}=e^{\hat{C}} \hat{H}_{v} e^{-\hat{C}}$ with $\hat{C}=\sum_{k} \lambda_{k}\left(\hat{b}_{k}-\hat{b}_{k}^{\dagger}\right) /\left(2 \omega_{k}\right)$, the light-matter coupling can be expressed through the dynamics of a quasispin $\hat{\Sigma}_{-}=|e\rangle\langle 1|$ coupled to a bath of bosons, governed by the Hamiltonian $\hat{H}_{\mathrm{SBM}}=\hat{H}_{\mathcal{S}}+\hat{H}_{v}^{\text {shift }}$ :

$$
\hat{H}_{\mathrm{SBM}}=\frac{\delta}{2} \hat{\Sigma}_{z}+\frac{g}{2} \hat{\Sigma}_{x}+\sum_{k}\left[\omega_{k} \hat{n}_{k}+\frac{\lambda_{k}}{2} \hat{\Sigma}_{z}\left(\hat{b}_{k}+\hat{b}_{k}^{\dagger}\right)\right] .
$$

Here, we have have introduced $|e\rangle=\hat{\sigma}^{+}|G\rangle$ and $|1\rangle=\hat{a}^{\dagger}|G\rangle$ as shortcuts for the excitonic and photonic states in the singleexcitation subspace, respectively.

The SBM constitutes one of the minimal models to study quantum dissipation in solid-state and organic systems, e.g., decoherence of quantum oscillations in qubits $[65,66]$, impurity moments coupled to bulk magnetic fluctuations [67], and electron transfer in biological molecules [68]. Here, the detuning between the cavity and the zero-phonon line $\delta=$ $\omega_{e}-\Delta-\omega_{O}$ plays the role of a bias, where the reorganization energy follows from Eq. (6).

The light-matter coupling $g$ favors the mixing of the molecular exciton and the photon, similarly to the HTC model analyzed in the previous section, whereas the spin-bath interaction dresses them with vibrational modes, destroying lightmatter coherence. In the unbiased case, i.e., when the cavity is resonant with the zero-phonon line $\omega_{O}=\omega_{e}-\Delta$, the Hamiltonian becomes parity symmetric under the exchange $|e\rangle \Leftrightarrow|1\rangle$. This symmetry is known to be spontaneously broken when the vibrational coupling becomes larger than a critical value for sub-Ohmic and Ohmic $J_{v}(\omega)$, leading to a quantum phase transition in which the ground state is a fully polarized spin state [69]. Translated to the present case, this would signify a vibrationally driven localization phenomenon in either excitonic or photonic states, precluding the formation of polaritons at the single-molecule limit. However, it is questionable whether traces of this transition could be observed in nanocavity systems capable of significant light-matter coupling in the single-molecule case $[10,70]$, especially considering that we are here neglecting the nonradiative and radiative losses associated with such systems. We thus have focused on the many-molecule case $(N>1)$ at zero detuning $\omega_{e}=$ $\omega_{O}$, corresponding to the most common experimental setups, while using the analogy to the SBM to make connections to the existing literature.
[1] A. S. Davydov, Theory of Molecular Excitons (Springer, Boston, 1971).

[2] V. M. Agranovich, Excitations in Organic Solids (Oxford University Press, Oxford, 2008), Vol. 142.

[3] S. Kéna-Cohen and S. R. Forrest, Nat. Photonics 4, 371 (2010).

[4] M. Ramezani, A. Halpin, A. I. Fernández-Domínguez, J. Feist, S. R.-K. Rodriguez, F. J. Garcia-Vidal, and J. Gómez Rivas, Optica 4, 31 (2017).

[5] E. Orgiu, J. George, J. A. Hutchison, E. Devaux, J. F. Dayen, B. Doudin, F. Stellacci, C. Genet, J. Schachenmayer, C. Genes, G. Pupillo, P. Samorì, and T. W. Ebbesen, Nat. Mater. 14, 1123 (2015)

[6] D. M. Coles, Y. Yang, Y. Wang, R. T. Grant, R. A. Taylor, S. K. Saikin, A. Aspuru-Guzik, D. G. Lidzey, J. K.-H. Tang, and J. M. Smith, Nat. Commun. 5, 5561 (2014).

[7] B. Munkhbat, M. Wersäll, D. G. Baranov, T. J. Antosiewicz, and T. Shegai, Sci. Adv. 4, eaas9552 (2018).
[8] D. G. Lidzey, D. D. C. Bradley, M. S. Skolnick, T. Virgili, S. Walker, and D. M. Whittaker, Nature (London) 395, 53 (1998).

[9] G. Zengin, M. Wersäll, S. Nilsson, T. J. Antosiewicz, M. Käll, and T. Shegai, Phys. Rev. Lett. 114, 157401 (2015).

[10] R. Chikkaraddy, B. de Nijs, F. Benz, S. J. Barrow, O. A. Scherman, E. Rosta, A. Demetriadou, P. Fox, O. Hess, and J. J. Baumberg, Nature (London) 535, 127 (2016).

[11] A. Kavokin and F. P. Laussy, Microcavities, Vol. 21 (Oxford University Press, Oxford, 2017).

[12] S. Pau, G. Björk, J. Jacobson, H. Cao, and Y. Yamamoto, Phys. Rev. B 51, 7090 (1995).

[13] F. Tassone, C. Piermarocchi, V. Savona, A. Quattropani, and P. Schwendimann, Phys. Rev. B 56, 7554 (1997).

[14] S. Pau, H. Cao, J. Jacobson, G. Björk, Y. Yamamoto, and A. Imamoğlu, Phys. Rev. A 54, R1789(R) (1996).

[15] S. Pau, G. Björk, J. Jacobson, H. Cao, and Y. Yamamoto, Phys. Rev. B 51, 14437 (1995). 
[16] F. Herrera and F. C. Spano, Phys. Rev. Lett. 116, 238301 (2016).

[17] J. Galego, F. J. Garcia-Vidal, and J. Feist, Nat. Commun. 7, 13841 (2016).

[18] P. Kirton and J. Keeling, Phys. Rev. Lett. 111, 100404 (2013).

[19] J. A. Ćwik, S. Reja, P. B. Littlewood, and J. Keeling, Europhys. Lett. 105, 47009 (2014).

[20] F. C. Spano, J. Chem. Phys. 142, 184707 (2015).

[21] M. A. Zeb, P. G. Kirton, and J. Keeling, ACS Photonics 5, 249 (2018).

[22] J. George, A. Shalabney, J. A. Hutchison, C. Genet, and T. W. Ebbesen, J. Phys. Chem. Lett. 6, 1027 (2015).

[23] T. K. Hakala, J. J. Toppari, A. Kuzyk, M. Pettersson, H. Tikkanen, H. Kunttu, and P. Törmä, Phys. Rev. Lett. 103, 053602 (2009).

[24] A. Nitzan, Chemical Dynamics in Condensed Phases: Relaxation, Transfer and Reactions in Condensed Molecular Systems, Oxford Graduate Texts (Oxford University Press Oxford, 2006), p. 752.

[25] N. Wu, J. Feist, and F. J. Garcia-Vidal, Phys. Rev. B 94, 195409 (2016).

[26] R. Orús, Ann Phys. (N.Y.) 349, 117 (2014).

[27] A. Weichselbaum, F. Verstraete, U. Schollwöck, J. I. Cirac, and J. von Delft, Phys. Rev. B 80, 165117 (2009).

[28] R. A. Marcus, J. Chem. Phys. 24, 966 (1956).

[29] G. D. Mahan, Many-particle Physics (Springer, Boston, 2000).

[30] J. del Pino, F. A. Schröder, A. W. Chin, J. Feist, and F. J. GarciaVidal, arXiv:1804.04511.

[31] J. Prior, A. W. Chin, S. F. Huelga, and M. B. Plenio, Phys. Rev. Lett. 105, 050404 (2010).

[32] A. W. Chin, S. F. Huelga, and M. B. Plenio, in Semiconductor Semimetals, Vol. 85 (Elsevier, Amsterdam, 2011), p. 115.

[33] A. Garg, J. N. Onuchic, and V. Ambegaokar, J. Chem. Phys. 83, 4491 (1985).

[34] M. Thoss, H. Wang, and W. H. Miller, J. Chem. Phys. 115, 2991 (2001).

[35] T. Renger and R. A. Marcus, J. Chem. Phys. 116, 9997 (2002).

[36] J. Iles-Smith, N. Lambert, and A. Nazir, Phys. Rev. A 90, 032114 (2014).

[37] D. M. Coles, A. J. H. M. Meijer, W. C. Tsoi, M. D. B. Charlton, J.-S. Kim, and D. G. Lidzey, J. Phys. Chem. A 114, 11920 (2010).

[38] A. P. Shreve, E. H. Haroz, S. M. Bachilo, R. B. Weisman, S. Tretiak, S. Kilina, and S. K. Doorn, Phys. Rev. Lett. 98, 037405 (2007).

[39] S. R. White, Phys. Rev. Lett. 69, 2863 (1992).

[40] S. Szalay, M. Pfeffer, V. Murg, G. Barcza, F. Verstraete, R. Schneider, and Ö. Legeza, Int. J. Quantum Chem. 115, 1342 (2015).

[41] Y.-Y. Shi, L.-M. Duan, and G. Vidal, Phys. Rev. A 74, 022320 (2006).

[42] F. A. Y. N. Schröder, D. H. P. Turban, A. J. Musser, N. D. M. Hine, and A. W. Chin, arXiv:1710.01362.
[43] C. Guo, A. Weichselbaum, J. von Delft, and M. Vojta, Phys. Rev. Lett. 108, 160401 (2012).

[44] F. A. Y. N. Schröder and A. W. Chin, Phys. Rev. B 93, 075105 (2016).

[45] F. Herrera and F. C. Spano, Phys. Rev. A 95, 053867 (2017).

[46] U. Weiss, in Quantum Dissipative Systems, edited by U. Weiss, Vol. 10 (World Scientific, Singapore, 2012).

[47] G. Zengin, G. Johansson, P. Johansson, T. J. Antosiewicz, M. Käll, and T. Shegai, Sci. Rep. 3, 3074 (2013).

[48] A. J. Leggett, S. Chakravarty, A. T. Dorsey, M. P. A. Fisher, A. Garg, and W. Zwerger, Rev. Mod. Phys. 59, 1 (1987).

[49] J. Galego, F. J. Garcia-Vidal, and J. Feist, Phys. Rev. X 5, 041022 (2015).

[50] S. Baieva, O. Hakamaa, G. Groenhof, T. T. Heikkilä, and J. J. Toppari, ACS Photonics 4, 28 (2017).

[51] P. E. Dargel, A. Wöllert, A. Honecker, I. P. McCulloch, U. Schollwöck, and T. Pruschke, Phys. Rev. B 85, 205119 (2012).

[52] S. Bera, A. Nazir, A. W. Chin, H. U. Baranger, and S. Florens, Phys. Rev. B 90, 075110 (2014).

[53] J. Eckel, J. H. Reina, and M. Thorwart, New J. Phys. 11, 085001 (2009).

[54] C. Gonzalez-Ballestero, F. A. Y. N. Schröder, and A. W. Chin, Phys. Rev. B 96, 115427 (2017).

[55] F. Valmorra, M. Bröll, S. Schwaiger, N. Welzel, D. Heitmann, and S. Mendach, Appl. Phys. Lett. 99, 051110 (2011).

[56] N. Christensson, B. Dietzek, A. Yartsev, and T. Pullerits, Vib. Spectrosc. 53, 2 (2010).

[57] S. A. Passino, Y. Nagasawa, and G. R. Fleming, J. Chem. Phys. 107, 6094 (1997).

[58] T. Schwartz, J. A. Hutchison, J. Léonard, C. Genet, S. Haacke, and T. W. Ebbesen, Chem. Phys. Chem. 14, 125 (2013).

[59] S. R. K. Rodriguez, J. Feist, M. A. Verschuuren, F. J. García Vidal, and J. Gómez Rivas, Phys. Rev. Lett. 111, 166802 (2013).

[60] A. W. Chin, J. Prior, R. Rosenbach, F. Caycedo-Soler, S. F. Huelga, and M. B. Plenio, Nat. Phys. 9, 113 (2013).

[61] U. Schollwöck, Ann. Phys. (N.Y.) 326, 96 (2011).

[62] J. Haegeman, C. Lubich, I. Oseledets, B. Vandereycken, and F. Verstraete, Phys. Rev. B 94, 165116 (2016).

[63] J. Haegeman, J. I. Cirac, T. J. Osborne, I. Pižorn, H. Verschelde, and F. Verstraete, Phys. Rev. Lett. 107, 070601 (2011).

[64] A. W. Chin, Á. Rivas, S. F. Huelga, and M. B. Plenio, J. Math. Phys. 51, 092109 (2010).

[65] T. A. Costi and R. H. McKenzie, Phys. Rev. A 68, 034301 (2003).

[66] D. V. Khveshchenko, Phys. Rev. B 69, 153311 (2004).

[67] S. Sachdev, C. Buragohain, and M. Vojta, Science 286, 2479 (1999).

[68] M. B. Plenio and S. F. Huelga, New J. Phys. 10, 113019 (2008).

[69] S. Sachdev, in Quantum Phase Transitions (Cambridge University Press, Cambridge, 2011).

[70] R. Liu, Z.-K. Zhou, Y.-C. Yu, T. Zhang, H. Wang, G. Liu, Y. Wei, H. Chen, and X.-H. Wang, Phys. Rev. Lett. 118, 237401 (2017). 